Article

\title{
The Zerotrope, a Dynamic Holographic Display: Design and Implementation
}

\author{
Philippe Gentet ${ }^{1} \mathbb{D}$, Jinbeom Joung ${ }^{1}$, Yves Gentet ${ }^{2}$, Kwang-Jib Kim ${ }^{1}$ and Seung-Hyun Lee ${ }^{3, *}{ }^{\mathbb{C}}$ \\ 1 Plasma Bio Display, Kwangwoon University, Seoul 01897, Korea \\ 2 Ultimate Holography, 33000 Bordeaux, France \\ 3 Ingenium College, Kwangwoon University, Seoul 01897, Korea \\ * Correspondence: shlee@kw.ac.kr; Tel.: +82-10-5285-5282
}

Received: 18 July 2019; Accepted: 12 August 2019; Published: 16 August 2019

\begin{abstract}
This paper presents the Zerotrope, an improved version of the classic phenakistiscope and zoetrope devices. This device is used to create a new 360-degree dynamic 3D display by the inclusion of a single ultra-realistic full-color hologram. The Zerotrope is built with a zero-degree transplane hologram mounted on a disk rotating at a constant speed. When a stroboscopic lamp synchronized with the rotation illuminates this hologram, the recorded characters, arranged radially around the center of the disk, are animated as in a stop-motion movie. The operation of the Zerotrope is successful and shows the effect of a 3D display without the need for special viewing aids.
\end{abstract}

Keywords: full-color hologram; 3D display; holographic display; $\mathrm{H} 2$ hologram

\section{Introduction}

Three-dimensional (3D) displays constitute an important research topic [1-3]. Most current approaches use the stereoscopic principle [4], which can cause visual discomfort and visual fatigue for the viewer [5]. Inspired by the pre-movie mechanical devices history, the authors designed the Fantatrope $^{\mathrm{TM}}$ in 2018. It is a holographic zoetrope composed of a series of twelve ultra-realistic full-color holograms arranged in a chronological sequence and mounted on a cylindrical rotating base [6]. A strobe light synchronized with the rotation speed successively illuminated the different frames and the recorded character was animated. The operation was successful and generated the effect of a real 3D display without the need for special glasses or other viewing aids. The purpose of this research was to build a second dynamic holographic display that was lighter and used a single hologram. The main inspiration for building this novel display was the phenakistiscope, the first device that created a fluid illusion of motion and was invented in 1833 by Joseph Plateau. The phenakistiscope comprises a spinning disc mounted vertically on a handle. A series of equidistant radial slots and pictures representing the phases of movement of a character are arranged around the circumference of the disk. The viewer rotates the disk by hand and views the sequence reflected in a mirror through the moving slots. Then, this rapid succession of pictures creates an illusion of a moving image for the viewer. However, this device demonstrates some limitations. As it is turned by hand, the rotation speed and the animation are not constant. Furthermore, the animation can be viewed by only one person at a time. Moreover, the user cannot observe the animation directly but only through a slot and a mirror. The earliest improvement of the phenakistiscope was the zoetrope, which was designed by William Horner [7] and gained popularity after 1867. The zoetrope is a cylinder with slots cut vertically on its sides and a band of paper consisting of a set of sequenced drawings or photographs on the inner surface. The illusion of motion is produced through a rapid succession of these static images observed through the slits. Modern 3D zoetropes exploit a principle similar to that of the traditional zoetrope, with three-dimensional (3D) solid models on a rotating turntable synchronized with a strobe light. 
These devices have attracted considerable interest in recent times due to the development of 3D printers. The 3D zoetrope demonstrates several improvements over the classical zoetrope. The speed rotation is constant and the animation is directly observable by several individuals. Moreover, the flashing strobe light produces clearer, sharper, and distortion-free results. However, this device also demonstrates some limitations. The animation needs to be observed in darkness to experience the strobe light effect. The 3D models need to be secured precisely on the turning table, and they can be fragile, heavy, and difficult to transport. This paper presents the Zerotrope ${ }^{\mathrm{TM}}$, which is an improved version of the classic phenakistiscope and 3D zoetrope, devices owing to the inclusion of a single ultra-realistic full-color hologram. The name Zerotrope includes "zero" (for zero-degree hologram) and the Greek-root word "tropos" (turning). This new 3D display retains the advantages of the previously described devices while addressing their limitations and improving the results. To build the Zerotrope, a zero-degree transplane hologram is recorded with a series of 3D characters arranged radially and horizontally around the center of the disk, as in a phenakistiscope. This hologram is then mounted on a rotating disk and illuminated with a synchronized strobe light as in a 3D zoetrope. All the principal functions of this new device can be regulated. This paper explains its construction and evaluates its performances. Portions of this work were presented at the SPIE Photonics West 2019 Conference during the Optical Design Challenge 2019, San Francisco, CA, USA [8].

\section{Materials and Methods}

\subsection{Materials for Recording Holograms}

The different holograms were recorded on iso-panchromatic silver-halide holographic material Ultimate 04 (U04) [9] glass plates. U04 is an emulsion designed for recording full-color analog holograms without any diffusion. The main advantage of U04 is its very high sensitivity $\left(200 \mu \mathrm{J} / \mathrm{cm}^{2}\right.$ per laser); U04 therefore requires only low-power lasers to record the holograms. For our experiments, the red wavelength was provided by a helium-neon laser $633 \mathrm{~nm} 20 \mathrm{~mW}$, the green was provided by a diode-pumped solid-state (DPSS) laser $532 \mathrm{~nm} 100 \mathrm{~mW}$, and the blue was provided by a DPSS $473 \mathrm{~nm}$ $50 \mathrm{~mW}$.

\subsection{Recording of Holograms $H 1$ and $H 2$}

The master hologram was recorded with the full-color single-beam reflection Denisyuk [10] method. This method enables the recording of ultra-realistic [11] holograms with a $180^{\circ}$ full-parallax. The RGB laser beams were combined, and their intensities were adjusted to $20 \mathrm{~mW}$ to obtain a uniform white laser beam. This beam was then transmitted into a beam expander and a spatial filter. The diverging beam obtained illuminates perfectly the model and holographic plate in a homogeneous way at an angle of zero-degree to the vertical of the plate (Figure 1).

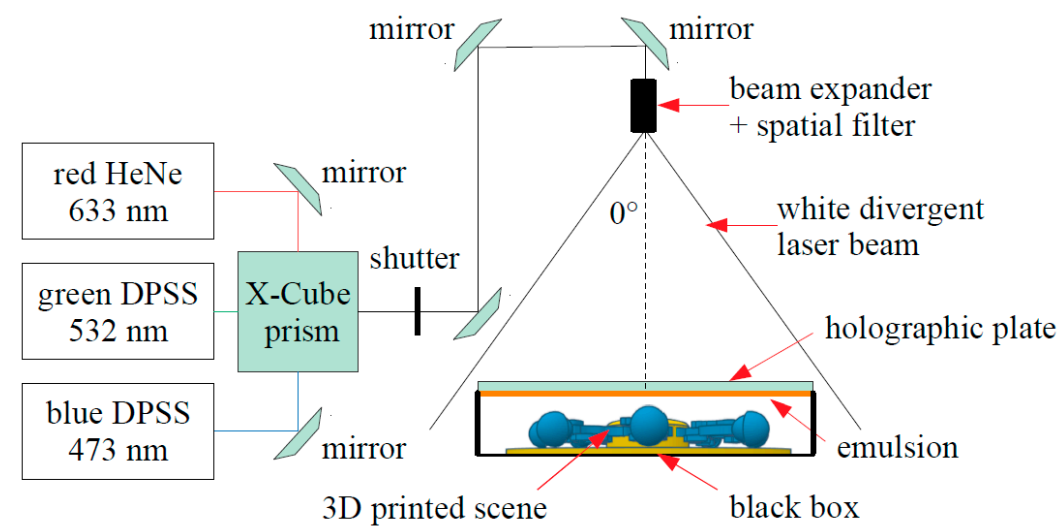

Figure 1. Zero-degree single-beam reflection Denisyuk hologram setup. 
Hologram H1 was then developed with two chemical baths (developer and bleach) as recommended by the manufacturer. To function as a master to record hologram H2, H1 must be reconstructed exactly with the same wavelengths used during the recording. Therefore, it was protected by a second glass plate and sealed with an optical ultraviolet (UV) glue to prevent any emulsion thickness variations (Figure 2).

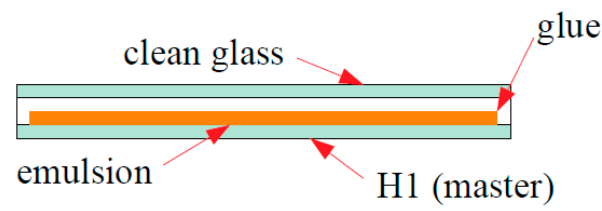

Figure 2. Hologram H1 is sealed with UV glue.

When hologram H1 was flipped, an inverted pseudoscopic real image was floating in front of the glass plate. The hologram $\mathrm{H} 2$ was then recorded with the same setup as before, but this time the flipped $\mathrm{H} 1$ acted as the model. $\mathrm{H} 2$ was developed according to the same recommended process and a floating orthoscopic real image was obtained. To be used in the Zerotrope, the final transplane image is expected to be completely outside and floating in front of the glass plate. Hologram $\mathrm{H} 2$ was then cut in the shape of a disk with a circular glass cutter and sealed with optical UV glue.

\subsection{Determining the H2 Oblique Field of View}

The oblique viewing angle of the zero-degree $\mathrm{H} 2$ hologram without visual distortion depends on the width $w(\mathrm{~m})$ of hologram $\mathrm{H} 1$ and the distance $d(\mathrm{~m})$ between the two holograms $\mathrm{H} 1$ and $\mathrm{H} 2$ during the copying process (Figure 3).

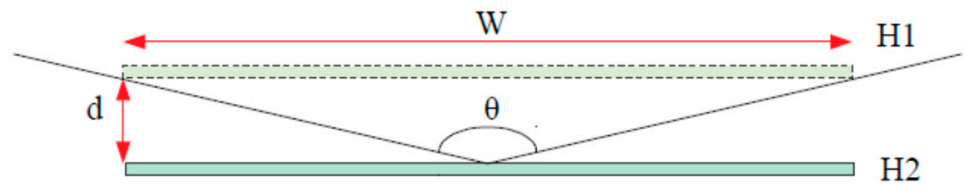

Figure 3. Hologram $\mathrm{H} 2$ oblique field of view without visual distortion.

The value of $\mathrm{H} 2$ hologram oblique field of view without visual distortion $\theta$ is calculated using the following expression:

$$
\theta=2 \arctan \frac{w}{2 d}
$$

\subsection{Generation of the 3D Content}

A series of equidistant radial 3D models representing the phases of movement of a character were horizontally arranged around the circumference of a disk. The complete 3D scene was designed with Autodesk's Tinkercad, a free online 3D design software, then printed by a fused deposition modeling (FDM) monochrome 3D printer with a white polylactic acid filament, and finally painted by hand.

\subsection{Animation of Hologram $\mathrm{H} 2$}

The $\mathrm{H} 2$ hologram was mounted on an acrylic disk of 12-cm diameter. A direct current (DC) gear motor was secured in a base and attached under the disk. This motor was rotated anticlockwise at a constant speed. To create the illusion of movement, a strobe light quickly flashed the hologram. A 3 W RGB light-emitting diode (LED) was placed $20 \mathrm{~cm}$ vertically above the center of the hologram at a zero-degree angle. A magnetic sensor was positioned under the disk and connected to a central processing unit (CPU) installed in the base. The CPU was used to control the rotation speed of the motor, background melody, and the flashes frequency and duration simultaneously. The device was powered by a battery. 


\subsection{Generation of a Sharp Image}

To obtain a sharp image without any motion blur, the stroboscopic light needs to freeze each element of the rotating disk. The stroboscopic parameters include the duration of the flash (or strobe rate), period between two flashes (or flash rate), and the intensity of each flash. The disk appears fixed and sharp when every point in the image travels a distance shorter than the resolving power of the human eye during the strobe rate [12]. The resolving power of a normal eye is $3 \times 10^{-4}$ radians. Therefore, for each specified distance between the disk and an observer, and for each rotation speed, there is a maximal strobe rate at which the observer cannot observe any blur in the image. If $\alpha$ (rad) is the resolving power of a normal eye, $D(\mathrm{~m})$ is the distance between the holographic disk and observer, $\omega(\mathrm{rad} / \mathrm{s})$ is the angular speed and $r(\mathrm{~m})$ is the radius. The maximal strobe rate $t(\mathrm{~s})$ is calculated using the following expression:

$$
t=\frac{\tan \alpha}{\omega r D}
$$

To solve the problems involving the motion blur, the CPU needs to be programmed to regulate the strobe rate according to Equation (2).

\section{Results and Discussion}

\section{1. $3 D$ Content and Holograms}

The computer-generated (CG) model consisted of a walking character showing eight sequential phases that were radially arranged around the center of a disk (Figure 4a). The disk was then 3D printed with $12 \mathrm{~cm}$ diameter and $1.5 \mathrm{~cm}$ thickness, and finally painted (Figure 4b). Hologram H1 of this 3D model was recorded with the single-beam reflection Denisyuk setup. A bright, colorful, and transparent virtual image was obtained (Figure 4c) with a $180^{\circ}$ full-parallax. This hologram H1 was then flipped and copied with the same holographic setup to obtain a transplane hologram $\mathrm{H} 2$ with similar brightness and transparency. Hologram $\mathrm{H} 2$ was completely outside, floating in front of the glass plate. $\mathrm{H} 2$ was then cut in the shape of a disk (Figure 4d) with a circular glass cutter and sealed with UV glue. For a viewer, there was no difference observed between watching the real 3D printed disk and its $\mathrm{H} 2$ hologram.

(a)

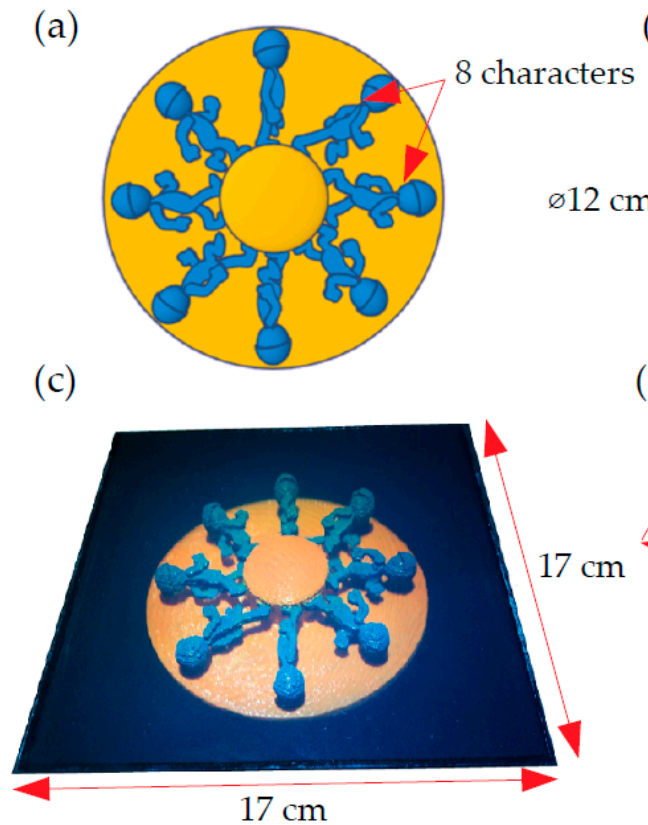

(b)

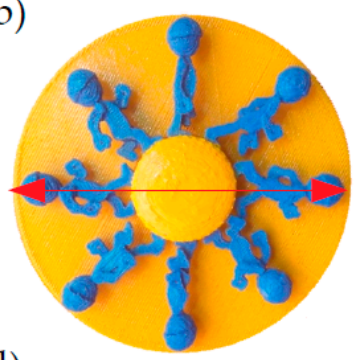

(d)
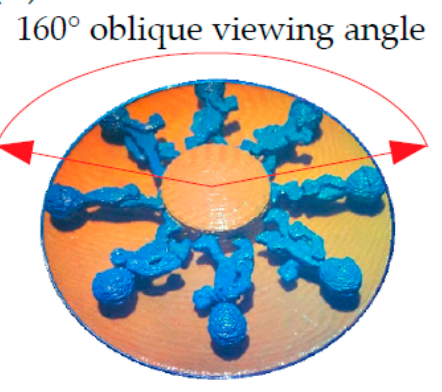

Figure 4. (a) Computer-generated model, (b) 3D printed and painted model, (c) H1 hologram, and (d) final floating $\mathrm{H} 2$. 
Given that the thickness of our model is $1.5 \mathrm{~cm}$ and the width of our holographic plate is $17 \mathrm{~cm}$, the oblique field of view of our final hologram $\mathrm{H} 2$ was approximately $160^{\circ}$ as derived using Equation (1). Observations confirmed this large oblique field of view without distortions. Beyond this angle, the image began to gradually disappear through the edges and deform. The use of silver-halide holographic glass plates has simplified the recording of different images. After processing, the resulting $\mathrm{H} 2$ was ultra-realistic and showed the "magic" of a true 3D image with a full-circle viewing of $360^{\circ}$ and a large oblique field of view without visual distortion.

\subsection{Motion Blur}

To obtain an image without motion blur, the CPU was programmed, according to Equation (2), to automatically regulate the duration of the flash based on the radius of the base $(0.06 \mathrm{~m})$, distance from the viewer $(0.5 \mathrm{~m})$, and the flash rate. The observations confirmed that there was no blur in the image irrespective of the rotation speed of the disk.

\subsection{Zerotrope Assembly and Operation}

The $\mathrm{H} 2$ hologram was mounted on the rotating acrylic disk of the Zerotrope. When the rotation speed increased and the hologram $\mathrm{H} 2$ was illuminated and reconstructed by the strobe RGB LED light at zero-degree, the elements recorded on the hologram appeared to the viewers as if they were all in motion. By varying the number of flashes per rotation, it was also possible to change the apparent direction of rotation. When the light flashed 8 times/turn, the character walks on the spot (Video S1). If the light flashed at a rate higher than 8 times/turn, the character walked forward (Video S2) and the animation became more fluid. If the rate was lower, the character walked backward (Video S3) and the animation became jerkier. The schematic of the Zerotrope assembly with the hologram $\mathrm{H} 2$ is shown in Figure 5.

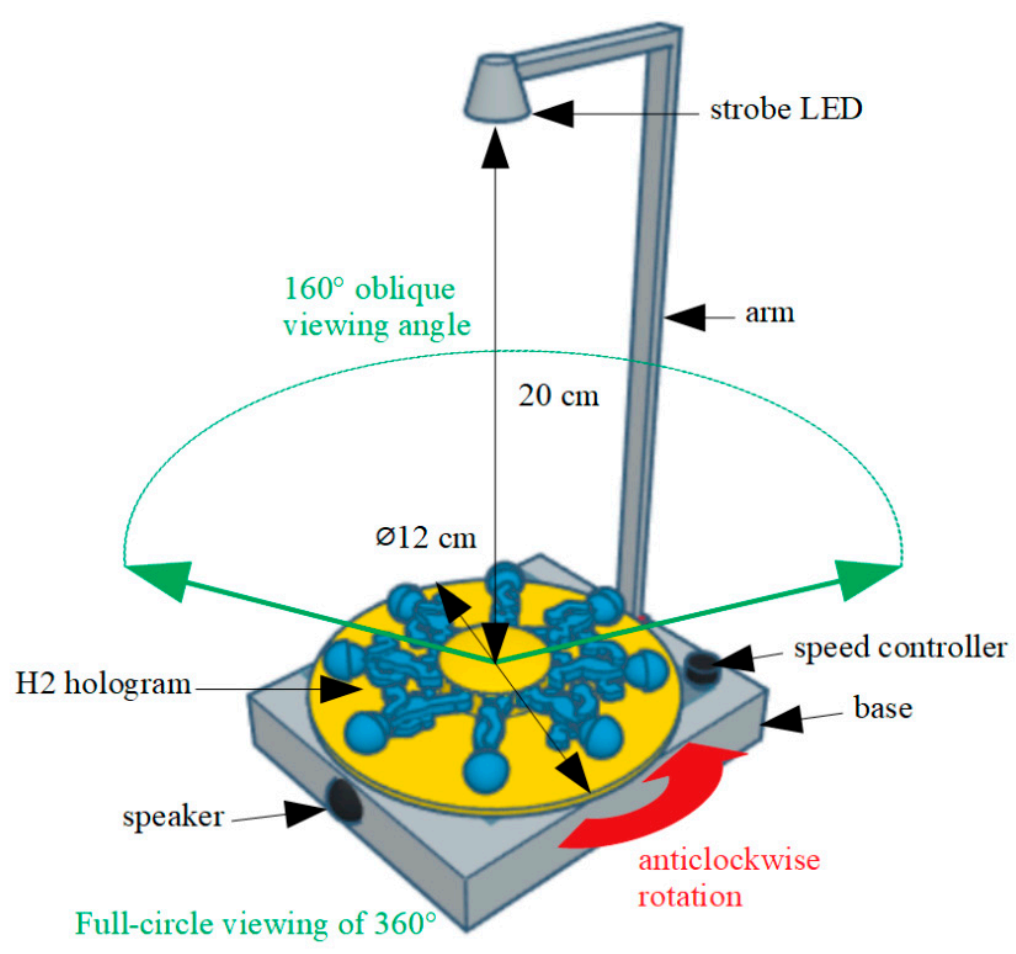

Figure 5. Schematic diagram for the proposed Zerotrope structure.

A constant rotation speed of $60 \mathrm{rpm}$ was sufficient to obtain a fluid motion of the characters. When the rotation speed increased, the strobe rate increased as well, and the flickering (visible luminance variation between images) decreased. We observed that the Zerotrope did not require a 
dark room to function as expected. Moreover, the flickering of the strobe light lessened due to the room illumination with ambient light at $150 \mathrm{lumen} / \mathrm{m}^{2}$ and no direct lighting other than the stroboscopic LED. The hologram could be attached and removed from the Zerotrope easily. In addition, the Zerotrope, which is powered by a battery, provides the advantages of easy assembly and application.

The Zerotrope has overcome the main defects of the classic phenakistiscope by producing an animation that is 3D, fluid, and observable by several users directly instead of through a slot. Similar to the 3D zoetrope, the Zerotrope could present all the elements placed on the disc in movement simultaneously. Additionally, it could be operated even in non-dark conditions.

The most important part of the Zerotrope is its floating H2 hologram. Getting this $\mathrm{H} 2$ with the master/copy Denisyuk technique is a long process that requires several steps. It primarily requires the creation of a real model in 3D and the recording of two holograms successively. The possibilities of animation are also limited. This solution could be enhanced by using a digital printed hologram (holoprint) instead of the Denisyuk analog hologram. The use of a 3D holographic printer (holoprinter) makes it possible to obtain a floating hologram directly from a CG model in a single step. This holoprint can be recorded at any size and present complex and fluid animations with gushing images and special visual effects impossible to get with a real model.

\section{Conclusions}

In this research, we have presented the construction of Zerotrope, a new and lightweight holographic display powered by a battery. This display needs a strobe light to function. The operation of the Zerotrope is successfully demonstrated and the effect of the holographic reality is described. This device uses a single transplane hologram and presents a short 3D cyclic animation in full-color that can be viewed without any special viewing aids. The holographic disks can be easily changed so that the Zerotrope presents different animations. Applications for this new device include entertainment (optical toy), promotional merchandise, and artistic creations.

Supplementary Materials: The following are available online at http://www.mdpi.com/2076-3417/9/16/3378/s1, Video S1: When the light flashed 8 times/turn, the character walks on the spot, Video S2: If the light flashed at a rate higher than 8 times/turn, the character walked forward, Video S3: If the light flashed at a rate lower than 8 times/turn, the character walked backward.

Author Contributions: P.G. conceived, designed and implemented the study. J.J. implemented the electronic and mechanical part, P.G. and Y.G. set up the holographic bench and recorded holograms. P.G. and K.-J.K. collected and analyzed field data. S.-H.L. coordinated the study. P.G. wrote the manuscript with input from all other authors who gave the final approval for publication.

Funding: This research was supported by the Ministry of Science and ICT (MSIT), Korea, under the Information Technology Research Center (ITRC) support program (IITP-2019-2015-0-00448) supervised by the Institute of Information \& Communications Technology Planning \& Evaluation.

Conflicts of Interest: The authors declare no conflict of interest. The funders had no role in the design of the study; in the collection, analyses, or interpretation of data; in the writing of the manuscript, or in the decision to publish the results.

\section{References}

1. Geng, J. Three-dimensional display technologies. Adv. Opt. Photonics 2013, 5, 456-535. [CrossRef] [PubMed]

2. Lim, Y.; Hong, K.; Kim, H.; Kim, H.E.; Chang, E.Y.; Lee, S.; Kim, T.; Nam, J.; Choo, H.G.; Kim, J.; et al. 360-degree tabletop electronic holographic display. Opt. Express 2016, 24, 24999-25009. [CrossRef] [PubMed]

3. Sugie, T.; Akamatsu, T.; Nishitsuji, T.; Hirayama, R.; Masuda, N.; Nakayama, H.; Ichihashi, Y.; Shiraki, A.; Oikawa, M.; Takada, N.; et al. High-performance parallel computing for next-generation holographic imaging. Nat. Electron. 2018, 1, 254-259. [CrossRef]

4. Ogle, K.N. Some aspects of stereoscopic depth perception. J. Opt. Soc. Am. 1967, 57, 1073-1081. [CrossRef] [PubMed]

5. Lambooij, M.; Fortuin, M.; Heynderickx, I.; IJsselsteijn, W. Visual diskomfort and visual fatigue of stereoscopic displays: A review. J. Imaging Sci. Technol. 2009, 53, 30201-1-30201-14. [CrossRef] 
6. Gentet, P.; Joung, J.; Gentet, Y.; Hamacher, A.; Lee, S.H. Fantatrope, a moving hologram display: Design and implementation. Opt. Express 2019, 27, 11571-11584. [CrossRef] [PubMed]

7. Horner, W.G. XI. On the properties of the Dædaleum, a new instrument of optical illusion. Philos. Mag. 1834, 4, 36-41. [CrossRef]

8. Gentet, P.; Joung, J.; Gentet, Y.; Lee, S.H. Design and implementation of the Zerotrope: A novel dynamic holographic display. In Proceedings of the Optical Design Challenge 2019 International Society for Optics and Photonics, San Francisco, CA, USA, 27 February 2019. [CrossRef]

9. Gentet, P.; Gentet, Y.; Lee, S.H. Ultimate 04 the new reference for ultra-realistic color holography. In Proceedings of the 2017 International Conference on Emerging Trends \& Innovation in ICT (ICEI), Pune, India, 3 February 2017; pp. 162-166.

10. Denisyuk, Y.N. On the reproduction of the optical properties of an object by the wave field of its scattered radiation. Opt. Spectrosc. Engl. Transl. 1965, 18, 152-157.

11. Bjelkhagen, H.; Brotherton-Ratcliffe, D. Ultra-Realistic Imaging: Advanced Techniques in Analogue and Digital Colour Holography; CRC Press: Boca Raton, FL, USA, 2016. [CrossRef]

12. Hartridge, H. Visual acuity and the resolving power of the eye. J. Physiol. 1922, 57, 52-67. [CrossRef] [PubMed]

(C) 2019 by the authors. Licensee MDPI, Basel, Switzerland. This article is an open access article distributed under the terms and conditions of the Creative Commons Attribution (CC BY) license (http://creativecommons.org/licenses/by/4.0/). 